P95 (continued)

College of Medicine; Suzanne Leson, PhD, LD, RDN, FAND, Ohio State University; Sotso Prieto Mercedes, PhD, Ohio State University, School of Applied Health Sciences and Wellness, College of Health Sciences and Professions; Michika Nickerson, MPH, MS, Ohio State University, Medical Dietetics, School of Health and Rehabilitation Sciences, College of Medicine

Background (Background, Rationale, Prior Research, and/or Theory): There is a discrepancy between African American women's (AAW) perception of weight versus actual weight. Further, AAW tend to have higher positive body image regardless of weight classification.

Objective: To examine the association between the different facets of body image and adiposity in AAW.

Study Design, Setting, Participants, Intervention: A cross-sectional study of 74 AAW residing in the Southeast U.S. were recruited. The four measures of the MBSRQAS, Appearance Evaluation (AE), Appearance orientation (AO), Overweight preoccupation (OP), and Self-classified weight (SCW), were used to measure body image. Body fat percentage (BFP) was used to measure adiposity.

Outcome Measures and Analysis: Measures of adiposity and body image. Ordinal regressions and odds ratios were examined.

Results: Mean percent body fat was 38.8 (SD 11.0). Means for the subscales were: AE (3.52 [SD .73]), AO (3.72, SD [.56]), OP (2.54 [SD .90]), and SCW (3.53 [SD .70]). Results indicated that there was a negative association between AE and BFP $(P<.001)$. For every $1 \%$ higher BFP there was an $8.4 \%$ lower odds that appearance would be rated favorably [OR $0.92,95 \%$ CI $(0.88,0.96)]$. SCW and OP were positively associated with BFP $(P<.05)$. For every $1 \%$ higher BFP there was a 19\% higher odds that participants would classify themselves as overweight and a 5\% higher odds, they would be preoccupied about being overweight. AO was not associated with BFP.

Conclusions and Implications: Our sample had high ratings of body image even though, on average, they were overweight. Interestingly, different facets of body image were differentially associated with BFP. The higher BFP, the more likely they were concerned with being overweight, classified themselves as overweight, and were less satisfied with their appearance. AAW at higher levels of BFP may be more willing to engage in healthy weight interventions due to their dissatisfaction with body image.

Funding: The African American Research and Studies Program.

\section{P96 Examination of Usefulness of a Meal Pattern Requirements Knowledge Assessment Tool}

Danya Johnson, BS, JohnsonDanyaL@uams.edu, University of Arkansas for Medical Sciences, 521 Jack Stephens Drive \#530, Little Rock, AR 72205; Leanne Whiteside-Mansell, EdD; Taren Swindle, PhD
Background (Background, Rationale, Prior Research, and/or Theory): Meal pattern requirements in the Child and Adult Care Food Program (CACFP) required for centers and day care homes are research-based and intended to support health and wellness for developing children. However, assessment of education to assure knowledge of the patterns is limited.

Objective: This study examined the psychometrics of a knowledge assessment of meal pattern requirements among child nutritional professionals.

Study Design, Setting, Participants, Intervention: Childcare nutrition professionals $(\mathrm{N}=267)$ in a southern rural state enrolled in free educational training focused on new meal pattern requirements completed pre-post assessment. Enrollees were administrators (50\%), cooks (25\%), or a combination (25\%). About half (47\%) had an associate degree or more education. Most (56\%) had more than 5 years of experience.

Outcome Measures and Analysis: Knowledge questions (9 pre and 20 post) were based on a training assessment created by the Institute of Child Nutrition. The nine items selected for administration both times targeted new regulations. Item analyses included an examination of item distribution. Pre/post regulations assessed include items related to serving juice, sugar, snacks, meat alternatives, and whole grain, in addition to use of formula for infants and food preparation. Change after education was assessed for items using McNemar's test and for the total correct score using pair t-tests.

Results: Most items (7/9) were scored incorrectly by over $25 \%$ of attendees at baseline. At post assessment, participants showed statistically significant knowledge gain on most (7/9) item and at least 75\% accurately scored the regulation on $17 / 20$ items. Pre-post test scores increased $14 \%$ $(\mathrm{M}=62 \%, \mathrm{SD}=20 \%$ to $\mathrm{M}=75 \%, \mathrm{SD}=14 \% ; \mathrm{t}(266)=$ $-10.8, P=.000)$. For the expanded assessment at posttest (20 items), the average score was $83 \%$ correct.

Conclusions and Implications: The tool demonstrated knowledge change before and after education. The short tool may be helpful in standardizing assessment across training to compare training quality.

Funding: USDA.

\section{P97 Examining Dinnertime Photos of Foods Served by Low-Income Families to Inform a Home Food Environment Checklist}

Savannah Hobbs, MEd, Colorado State University; Morgan McCloskey, MSPH; Susan L. Johnson, PhD, University of Colorado, Anschutz Medical Campus; Richard E. Boles, PhD; Laura L. Bellows, PhD, MPH, RD, laura.bellows@colostate.edu, Colorado State University, Department FSHN, 1571 Campus Delivery, Fort Collins, CO 80523-1571

Background (Background, Rationale, Prior Research, and/or Theory): The home food environment (HFE) plays an important role in food preferences, habits, and health as it encompasses foods recommended for a 